\title{
A NEW CHARACTERIZATION OF SUBMANIFOLDS WITH PARALLEL MEAN CURVATURE VECTOR IN $S^{n+p}$
}

\author{
Abdênago Alves de Barros, Aldir Chaves Brasil Jr. AND \\ Luis Amancio Machado de Sousa JR.
}

\begin{abstract}
In this work we will consider compact submanifold $M^{n}$ immersed in the Euclidean sphere $S^{n+p}$ with parallel mean curvature vector and we introduce a Schrödinger operator $L=-\Delta+V$, where $\Delta$ stands for the Laplacian whereas $V$ is some potential on $M^{n}$ which depends on $n, p$ and $h$ that are respectively, the dimension, codimension and mean curvature vector of $M^{n}$. We will present a gap estimate for the first eigenvalue $\mu_{1}$ of $L$, by showing that either $\mu_{1}=0$ or $\mu_{1} \leq-n\left(1+H^{2}\right)$. As a consequence we obtain new characterizations of spheres, Clifford tori and Veronese surfaces that extend a work due to $\mathrm{Wu}[\mathrm{W}]$ for minimal submanifolds.
\end{abstract}

\section{Introduction}

Let $M^{n}$ be a closed Riemannian manifold, i.e. $M^{n}$ is compact without boundary, and denote by $S^{n+p}$ the Euclidean sphere of sectional curvature one. For an immersion $\psi: M^{n} \rightarrow S^{n+p}$ we will denote by $A$ its second fundamental form whereas $h$ stands for its mean curvature vector and the mean curvature is defined by $H=|h|$. We introduce on $M^{n}$ the traceless tensor $\Phi=A-h g$, where $g$ stands for the induced metric on $M$ and we consider $\Phi_{h}(X, Y)=\langle\Phi(X, Y), h\rangle$ for any tangent vector fields $X, Y$ on $M^{n}$. It is easy to check that $|\Phi|^{2}=|A|^{2}-$ $n H^{2}$. Moreover, $|\Phi|^{2}=0$ if, and only if, $\psi\left(M^{n}\right)$ is totally umbilic. Now we define constants $B_{p, h}$ and $\rho=\rho(n, p, h)$ as follows

$$
B_{p, h}=\left\{\begin{array}{l}
\frac{1}{(2-1 / p)}, \quad \text { if } p=1 \text { or } h=0 \\
\frac{1}{(2-1 /(p-1))}, \quad \text { if } p \neq 1
\end{array}\right.
$$

and

$$
\rho=B_{p, h}\left\{n\left(1+H^{2}\right)-\frac{n(n-2)}{\sqrt{n(n-1)}}\left|\Phi_{h}\right|\right\} .
$$

1991 Mathematics Subject Classification: [2000] Primary 53C42, 53A10.

Key words and phrases: Mean curvature vector, first eigenvalue, Clifford torus.

We would like to thank FINEP and FAPERJ for financial support.

Received November 11, 2002; revised August 19, 2003. 
When $\psi: M^{n} \rightarrow S^{n+1}$ is a hypersurface H. Alencar and M. do Carmo [AC] have classified tori with constant mean curvature such that $|\Phi|^{2} \leq \rho$. They work was inspired by the ideas of the earlier papers due to J. Simons [Si], S. S. Chern, M. do Carmo and S. Kobayashi $[\mathrm{CdCK}]$ and B. Lawson [L]. For codimension bigger than one, supposing in addition that $h$ is a parallel vector, W. Santos ([S], p. 405) and $\mathrm{H} . \mathrm{Xu}([\mathrm{X}]$, p. 494) have generalized, independently, the work due to H. Alencar and M. do Carmo by showing that $|\Phi|^{2} \leq \rho$ implies either $|\Phi|^{2}=0$ or $|\Phi|^{2}=\rho$. Moreover, they have described all such $M^{n}$ by showing that $M^{n}$ is a sphere in the first case and either one of the Clifford tori or one of the Veronese surface in the second case. On the other hand, introducing the Schrödinger operator

$$
L=-\Delta-\left(2-\frac{1}{p}\right)|A|^{2},
$$

where $\Delta$ stands for the Laplacian on $M^{n}, \mathrm{C} . \mathrm{Wu}[\mathrm{W}]$ has proved the following result concerning a minimal submanifold of $S^{n+p}$.

THEOREM $1[\mathrm{C} . \mathrm{Wu}] . \quad$ Let $M^{n}$ be an n-dimensional closed minimally immersed submanifold in a unit sphere $S^{n+p}$ and let $\mu_{1}$ be the first eigenvalue of $L$. If $\mu_{1} \geq-n$ then either $\mu_{1}=0$ and $M^{n}$ is totally geodesic, or $\mu_{1}=-n$ and $M^{n}$ is the Veronese surface in $S^{4}$ or the Clifford torus in $S^{n+1}$.

The purpose of this paper is to extend the above result for closed submanifold in a unit sphere with parallel mean curvature vector. Before announcing our main result one introduces the following operator:

$$
L_{2}=-\Delta-B_{p, h}^{-1}|\Phi|^{2}-\frac{n(n-2)}{\sqrt{n(n-1)}}\left|\Phi_{h}\right| .
$$

We point out that $H=0$ yields $L_{2}=L$, where $L$ is the operator considered by $\mathrm{C}$. Wu on $[\mathrm{W}]$. Taking into account this fact we generalize Wu's result according to the following theorem:

THEOREM 2. Let $M^{n}$ be a closed submanifold of $S^{n+p}$ with mean curvature vector $h$ parallel and let $\mu_{1}$ be the first eigenvalue of $L_{2}$. Then either $\mu_{1}=0$ and $M^{n}$ is totally umbilic, or $\mu_{1} \leq-n\left(1+H^{2}\right)$. Moreover, $\mu_{1}=-n\left(1+H^{2}\right)$ if, and only if, $|\Phi|^{2}=\rho$; in this case $M^{n}$ is either the Veronese surface or the Clifford torus.

\section{Preliminaries}

Throughout this section we will introduce some basic facts and notations that will appear on this paper. A Riemannian manifold of dimension $k$ will be denoted by $M^{k}$. Now let $M^{n}$ be a closed submanifold immersed in a unit Euclidean sphere $S^{n+p}$. We use the following standard convention of index: 


$$
1 \leq A, B, C, \ldots, \leq n+p, 1 \leq i, j, k, \ldots, \leq n, n+1 \leq \alpha, \beta, \gamma, \ldots, \leq n+p .
$$

We consider an adapted orthonormal local frame $\left\{e_{A}\right\}$ and its associated connection forms $\left\{\omega_{A}\right\}$ on $S^{n+p}$. Restricting those forms to $M$ we get

$$
\begin{aligned}
& \omega_{\alpha i}=\sum_{j} h_{i j}^{\alpha} \omega_{j}, h_{i j}^{\alpha}=h_{j i}^{\alpha}, \\
& A=\sum_{i, j, \alpha} h_{i j}^{\alpha} \omega_{i} \otimes \omega_{j} \otimes \omega_{\alpha}
\end{aligned}
$$

and

$$
h=\frac{1}{n} \sum_{i, \alpha} h_{i i}^{\alpha} e_{\alpha} .
$$

If $R_{i j k l}$ and $R_{\alpha \beta \beta l}$ stand for the tensor of curvature and normal curvature, respectively, then Gauss, Ricci and Codazzi equations can be read, respectively, as follows:

$$
\begin{gathered}
R_{i j k l}=\delta_{i k} \delta_{j l}-\delta_{i l} \delta_{j k}+\sum_{\alpha}\left(h_{i k}^{\alpha} h_{j l}^{\alpha}-h_{i l}^{\alpha} h_{j k}^{\alpha}\right) \\
R_{\alpha \beta k l}=\sum_{i, j}\left(h_{i k}^{\alpha} h_{j l}^{\beta}-h_{i l}^{\alpha} h_{j k}^{\beta}\right)
\end{gathered}
$$

and

$$
h_{i j k}^{\alpha}=h_{i k j}^{\alpha}
$$

On the other hand, the traceless tensor $\Phi$ previously considered can be given by

$$
\Phi=\sum_{i, j, \alpha} \Phi_{i j}^{\alpha} \omega_{i} \otimes \omega_{j} \otimes e_{\alpha},
$$

where $\Phi_{i j}^{\alpha}=h_{i j}^{\alpha}-(1 / n) \operatorname{tr} H_{\alpha} \delta_{i j}$ and $H_{\alpha}=\left(h_{i j}^{\alpha}\right)$. Denoting by $N(T)$ the squared of the norm of a symmetric operator $T$, we have $N(A)=|A|^{2}=\sum_{i, j, \alpha}\left(h_{i j}^{\alpha}\right)^{2}$, whereas $N(\Phi)=|\Phi|^{2}=\sum_{\alpha} \operatorname{tr}\left(\Phi_{\alpha}^{2}\right)=|A|^{2}-n H^{2}$. We note also that the gradient of $\Phi$, denoted by $\nabla \Phi$, verifies

$$
|\nabla \Phi|^{2}=\sum_{i, j, \alpha}\left|\nabla \Phi_{i j}^{\alpha}\right|^{2}=\sum_{i, j, k, \alpha}\left(\Phi_{i j k}^{\alpha}\right)^{2},
$$

while the gradient of $|\Phi|^{2}$ satisfies the following identity:

$$
\left.\left.|\nabla| \Phi\right|^{2}\right|^{2}=4 \sum_{k}\left(\sum_{i, j, \alpha} \Phi_{i j}^{\alpha} \Phi_{i j k}^{\alpha}\right)^{2}
$$



gives

We will use also the following notation $\langle\Delta \Phi, \Phi\rangle=\sum_{i, j, \alpha} \Phi_{i j}^{\alpha} \Delta \Phi_{i j}^{\alpha}$, which

$$
\frac{1}{2} \Delta|\Phi|^{2}=\langle\Delta \Phi, \Phi\rangle+|\nabla \Phi|^{2}
$$

\section{Proof of Theorem 2}

In order to show our theorem we will need some auxiliary results. At first we will show two general lemmas. The first one can be read as follows:

LEMMA 1. Let $M^{n}$ be a Riemannian manifold isometrically immersed into a Riemannian manifold $N^{n+p}$. Consider $\Psi=\sum_{i, j, \alpha} \Psi_{i j}^{\alpha} \omega_{i} \otimes \omega_{j} \otimes e_{\alpha}$ a traceless symmetric tensor satisfying Codazzi equation. Then the following inequality holds

$$
\left.\left.|\nabla| \Psi\right|^{2}\right|^{2} \leq \frac{4 n}{(n+2)}|\Psi|^{2}|\nabla \Psi|^{2}
$$

where $|\Psi|^{2}=\sum_{i, j, \alpha}\left(\Psi_{i j}^{\alpha}\right)^{2}$ and $|\nabla \Psi|^{2}=\sum_{i, j, k, \alpha}\left(\Psi_{i j k}^{\alpha}\right)^{2}$. In particular the conclusion holds for the tensor $\Phi$ defined in the introduction.

Proof. First we fix $e_{\alpha}$ and define $\Psi^{\alpha}$ as the $\alpha$-component of $\Psi$. Now we take an orthonormal frame $\left\{e_{i}^{\alpha}\right\}$ of eigenfunctions of $\Psi^{\alpha}$ with correspondent eigenvalues $\mu_{i}^{\alpha}$. Hence we have

$$
\left.\left.|\nabla| \Psi^{\alpha}\right|^{2}\right|^{2}=4 \sum_{k}\left(\sum_{i, j} \Psi_{i j}^{\alpha} \Psi_{i j k}^{\alpha}\right)^{2}=4 \sum_{k}\left(\sum_{i} \mu_{i}^{\alpha} \Psi_{i i k}^{\alpha}\right)^{2} .
$$

By using Cauchy-Schwarz inequality we have

$$
\left.\left.|\nabla| \Psi^{\alpha}\right|^{2}\right|^{2} \leq 4 \sum_{i}\left(\mu_{i}^{\alpha}\right)^{2} \sum_{i, k}\left(\Psi_{i i k}^{\alpha}\right)^{2} .
$$

This can be rewritten as

$$
4\left|\Psi^{\alpha}\right|^{2}\left(\sum_{i}\left(\Psi_{i i i}^{\alpha}\right)^{2}+\sum_{i, k, i \neq k}\left(\Psi_{i i k}^{\alpha}\right)^{2}\right) \geq\left.\left.|\nabla| \Psi^{\alpha}\right|^{2}\right|^{2} .
$$

Now we fix an index $i$. Taking into account that $\operatorname{tr}\left(\Psi^{\alpha}\right)=0$, we conclude that $\Psi_{i i i}^{\alpha}=-\sum_{k, k \neq i} \Psi_{k k i}^{\alpha}$. By using Cauchy-Schwarz inequality again we have

$$
\sum_{i}\left(\Psi_{i i i}^{\alpha}\right)^{2}=\sum_{i}\left(\sum_{k, k \neq i} \Psi_{k k i}^{\alpha}\right)^{2} \leq(n-1) \sum_{k, i, k \neq i}\left(\Psi_{i i k}^{\alpha}\right)^{2} .
$$

Hence we obtain from inequalities (3.2) and (3.3) that

$$
\left.\left.|\nabla| \Psi^{\alpha}\right|^{2}\right|^{2} \leq 4 n\left|\Psi^{\alpha}\right|^{2} \sum_{i, k, i \neq k}\left(\Psi_{i i k}^{\alpha}\right)^{2} .
$$


On the other hand $\Psi_{i k}^{\alpha}=\Psi_{k i}^{\alpha}$ implies $\Psi_{i k i}^{\alpha}=\Psi_{k i i}^{\alpha}$. In view of Codazzi equation we obtain

$$
\Psi_{i i k}^{\alpha}=\Psi_{i k i}^{\alpha}=\Psi_{k i i}^{\alpha} .
$$

Since $\left|\nabla \Psi^{\alpha}\right|^{2}=\sum_{i, j, k}\left(\Psi_{i j k}^{\alpha}\right)^{2}$ and

$$
\sum_{i, j, k}\left(\Psi_{i j k}^{\alpha}\right)^{2}=\sum_{i}\left(\Psi_{i i i}^{\alpha}\right)^{2}+\sum_{i, k ; i \neq k}\left(\left(\Psi_{i i k}^{\alpha}\right)^{2}+\left(\Psi_{i k i}^{\alpha}\right)^{2}+\left(\Psi_{k i i}^{\alpha}\right)^{2}\right)+6 \sum_{i<j<k}\left(\Psi_{i j k}^{\alpha}\right)^{2}
$$

we may use (3.5) to conclude

$$
\left|\Psi^{\alpha}\right|^{2}\left|\nabla \Psi^{\alpha}\right|^{2}=\left|\Psi^{\alpha}\right|^{2}\left(\sum_{i}\left(\Psi_{i i i}^{\alpha}\right)^{2}+3 \sum_{i, k, i \neq k}\left(\Psi_{i i k}^{\alpha}\right)^{2}+6 \sum_{i<j<k}\left(\Psi_{i j k}^{\alpha}\right)^{2}\right) .
$$

It follows from this last equation the next inequality

$$
\left|\Psi^{\alpha}\right|^{2}\left|\nabla \Psi^{\alpha}\right|^{2} \geq 2\left|\Psi^{\alpha}\right|^{2} \sum_{i, k, i \neq k}\left(\Psi_{i i k}^{\alpha}\right)^{2}+\left|\Psi^{\alpha}\right|^{2}\left(\sum_{i, k, i \neq k}\left(\Psi_{i i k}^{\alpha}\right)^{2}+\sum_{i}\left(\Psi_{i i i}^{\alpha}\right)^{2}\right) .
$$

Combining the first term of the right hand side of this last inequality with (3.4) and the second term with (3.2) we derive

$$
\left|\Psi^{\alpha}\right|^{2}\left|\nabla \Psi^{\alpha}\right|^{2} \geq\left.\left.\frac{1}{2 n}|\nabla| \Psi^{\alpha}\right|^{2}\right|^{2}+\left.\left.\frac{1}{4}|\nabla| \Psi^{\alpha}\right|^{2}\right|^{2} .
$$

Since $\left.\left.|\nabla| \Psi\right|^{2}\right|^{2}=\left.\left.\sum_{\alpha}|\nabla| \Psi^{\alpha}\right|^{2}\right|^{2}$ and $\left|\Psi^{\alpha}\right|^{2} \leq|\Psi|^{2}$ it follows from the last inequality that

$$
\left.\left.|\nabla| \Psi\right|^{2}\right|^{2} \leq \frac{4 n}{n+2}|\Psi|^{2}|\nabla \Psi|^{2},
$$

which finishes the proof of the Lemma 1.

Now we consider the differentiable function $f_{\varepsilon}=\left(|\Phi|^{2}+\varepsilon\right)^{1 / 2}$ defined on $M^{n}$, where $\Phi$ is the traceless tensor previously defined in the introduction, $\varepsilon$ is a positive number and we prove the following lemma concerning this function.

Lemma 2. Let $M^{n}$ be a Riemannian manifold immersed in $S^{n+p}$ and let $f_{\varepsilon}$ be the function above defined. Then the Laplacian of $f_{\varepsilon}$ satisfies the inequality

$$
f_{\varepsilon} \Delta f_{\varepsilon} \geq \frac{2\left(|\Phi|^{2}+\varepsilon\right)^{-1}}{(n+2)}|\Phi|^{2}|\nabla \Phi|^{2}+\langle\Delta \Phi, \Phi\rangle .
$$

Proof. Since $\Delta f_{\varepsilon}=\operatorname{div}\left(\nabla f_{\varepsilon}\right)$ and $\nabla f_{\varepsilon}=\left(\left(|\Phi|^{2}+\varepsilon\right)^{-1 / 2} / 2\right)\left(\nabla|\Phi|^{2}\right)$ we have

$$
f_{\varepsilon} \Delta f_{\varepsilon}=\frac{1}{2} \Delta|\Phi|^{2}-\frac{\left(|\Phi|^{2}+\varepsilon\right)^{-1}}{4}\left\langle\nabla|\Phi|^{2}, \nabla|\Phi|^{2}\right\rangle .
$$


Using (2.9), we may conclude

$$
f_{\varepsilon} \Delta f_{\varepsilon}=\left(|\Phi|^{2}+\varepsilon\right)^{-1}\left(|\nabla \Phi|^{2}\left(|\Phi|^{2}+\varepsilon\right)-\left.\left.\frac{1}{4}|\nabla| \Phi\right|^{2}\right|^{2}\right)+\langle\Delta \Phi, \Phi\rangle .
$$

On the other hand, Lemma 1 yields $\left.\left.(1 / 4)|\nabla| \Phi\right|^{2}\right|^{2} \leq(n /(n+2))|\Phi|^{2}|\nabla \Phi|^{2}$. Since $|\Phi|^{2}+\varepsilon \geq|\Phi|^{2}$ we have

$$
\left(|\nabla \Phi|^{2}\left(|\Phi|^{2}+\varepsilon\right)-\left.\left.\frac{1}{4}|\nabla| \Phi\right|^{2}\right|^{2}\right) \geq|\Phi|^{2}|\nabla \Phi|^{2}\left(1-\frac{n}{(n+2)}\right),
$$

that is

$$
\left(|\nabla \Phi|^{2}\left(|\Phi|^{2}+\varepsilon\right)-\left.\left.\frac{1}{4}|\nabla| \Phi\right|^{2}\right|^{2}\right) \geq \frac{2|\Phi|^{2}|\nabla \Phi|^{2}}{(n+2)}
$$

Putting together equations (3.6) and (3.7) we have

$$
f_{\varepsilon} \Delta f_{\varepsilon} \geq \frac{2\left(|\Phi|^{2}+\varepsilon\right)^{-1}}{(n+2)}\left(|\Phi|^{2}|\nabla \Phi|^{2}\right)+\langle\Delta \Phi, \Phi\rangle,
$$

which finishes the proof of the Lemma 2.

Now let $L_{2}$ be the Schrödinger operator considered in the introduction. We will prove the next proposition concerning to the first eigenvalue of $L_{2}$, which extends a result derived by $\mathrm{C}$. $\mathrm{Wu}[\mathrm{W}]$ in the minimal case.

Proposition 1. Let $M^{n}$ be a closed submanifold immersed in $S^{n+p}$ with parallel mean curvature vector $h$ in such way that $M^{n}$ is not totally umbilic. If $\mu_{1}$ is the first eigenvalue of $L_{2}$ then

$$
\mu_{1} \leq-n\left(1+H^{2}\right)-\frac{2}{(n+2)} \frac{\int_{M}|\nabla \Phi|^{2} * 1}{\int_{M}|\Phi|^{2} * 1},
$$

where $* 1$ stands for the form of volume of $M^{n}$.

Proof. If we define the set $\Gamma=\left\{f \in C^{\infty}(M): f \neq 0\right\}$ then Rayleigh quotient yields $\mu_{1}=\inf _{f \in \Gamma}\left(\int_{M} f L_{2} f * 1 / \int_{M} f^{2} * 1\right)$. We consider now $f_{\varepsilon}=\left(|\Phi|^{2}+\right.$ $\varepsilon)^{1 / 2}$ the differentiable function given in the previous lemma. Since $M^{n}$ is not totally umbilic we get $\lim _{\varepsilon \rightarrow 0} \int_{M} f_{\varepsilon}^{2} * 1=\int_{M}|\Phi|^{2} * 1>0$. Thus we may use $f_{\varepsilon}$ as a test function to compute $\mu_{1}$. On the other hand, since $h$ is parallel W. Santos ([S], p. 405) has showed the following inequality

$$
\langle\Delta \Phi, \Phi\rangle \geq \Lambda|\Phi|^{2},
$$

where $\Lambda=\left\{n\left(1+H^{2}\right)-(n(n-2) / \sqrt{n(n-1)})\left|\Phi_{h}\right|-B_{p, h}^{-1}|\Phi|^{2}\right\}$.

Therefore we may combine Lemma (2) and inequality (3.8) to obtain

$$
f_{\varepsilon} \Delta f_{\varepsilon} \geq \frac{2}{(n+2)}\left(|\Phi|^{2}+\varepsilon\right)^{-1}|\Phi|^{2}|\nabla \Phi|^{2}+\Lambda|\Phi|^{2} .
$$


Since $f_{\varepsilon} L_{2} f_{\varepsilon}=-f_{\varepsilon} \Delta f_{\varepsilon}-B_{p, h}^{-1}|\Phi|^{2} f_{\varepsilon}^{2}-(n(n-2) / \sqrt{n(n-1)})\left|\Phi_{h}\right| f_{\varepsilon}^{2}$, we get

$$
\begin{aligned}
f_{\varepsilon} L_{2} f_{\varepsilon} \leq & -\frac{2\left(|\Phi|^{2}+\varepsilon\right)^{-1}\left(|\Phi|^{2}|\nabla \Phi|^{2}\right)}{(n+2)}-n\left(1+H^{2}\right)|\Phi|^{2} \\
& +\frac{n(n-2)|\Phi|^{2}\left|\Phi_{h}\right|}{\sqrt{n(n-1)}}+B_{p, h}^{-1}\left(|\Phi|^{2}\right)^{2} \\
& -\frac{n(n-2)\left|\Phi_{h}\right|}{\sqrt{n(n-1)}}\left(|\Phi|^{2}+\varepsilon\right)-B_{p, h}^{-1}\left(|\Phi|^{2}+\varepsilon\right)|\Phi|^{2} .
\end{aligned}
$$

From where we obtain

$$
f_{\varepsilon} L_{2} f_{\varepsilon} \leq-n\left(1+H^{2}\right)|\Phi|^{2}-\frac{2\left(|\Phi|^{2}+\varepsilon\right)^{-1}}{(n+2)}|\Phi|^{2}|\nabla \Phi|^{2} .
$$

Since $\mu_{1} \leq\left(\int_{M} f_{\varepsilon} L_{2} f_{\varepsilon} * 1 / \int_{M} f_{\varepsilon}^{2} * 1\right)$ and $H$ is constant inequality (3.10) yields

$$
\mu_{1} \leq-n\left(1+H^{2}\right) \frac{\int_{M}|\Phi|^{2} * 1}{\int_{M}\left(|\Phi|^{2}+\varepsilon\right) * 1}-\frac{2}{(n+2)} \frac{\int_{M}\left(|\Phi|^{2}+\varepsilon\right)^{-1}|\Phi|^{2}|\nabla \Phi|^{2} * 1}{\int_{M}\left(|\Phi|^{2}+\varepsilon\right) * 1} .
$$

Making $\varepsilon \rightarrow 0$ on the last inequality, we obtain

$$
\mu_{1} \leq-n\left(1+H^{2}\right)-\frac{2}{(n+2)} \frac{\int_{M}|\nabla \Phi|^{2} * 1}{\int_{M}|\Phi|^{2} * 1},
$$

which completes the proof of the desired result.

On the next proposition we consider the case when $M^{n}$ is not pseudoumbilical. By a pseudo-umbilical submanifold $M^{n}$ into $S^{n+p}$ we mean that $h$ is an umbilic direction of the second fundamental form $A$ of $M^{n}$. Now let $L_{3}=-\Delta-(n / 2 \sqrt{n-1})|A|^{2}$ be a new Schrödinger operator and let us prove an estimate concerning to its first eigenvalue according to the following proposition.

Proposition 2. Let $M^{n}$ be a closed submanifold immersed in $S^{n+p}$ with parallel non null mean curvature vector $h$ in such way that $M^{n}$ is not pseudoumbilical. If $\mu_{1}$ is the first eigenvalue of $L_{3}$ then

$$
\mu_{1} \leq-n-\frac{2}{(n+2)} \frac{\int_{M}\left|\nabla \Phi^{n+1}\right|^{2} * 1}{\int_{M}\left|\Phi^{n+1}\right|^{2} * 1}
$$

where $\Phi^{n+1}=\Phi_{i j}^{n+1} e_{n+1}, \Phi_{i j}^{n+1}=\left(h_{i j}^{n+1}-H \delta_{i j}\right)$ and $e_{n+1}=h / H$.

Proof. The proof is similar to the previous proposition. Indeed, let us consider $\left|\nabla\left(\Phi^{n+1}\right)\right|^{2}=\sum_{i, j, k}\left(\Phi_{i j k}^{n+1}\right)^{2}$ and $\left\langle\Delta\left(\Phi^{n+1}\right), \Phi^{n+1}\right\rangle=\sum_{i, j} \Phi_{i j}^{n+1} \Delta \Phi_{i j}^{n+1}$. Now it is enough to define $g_{\varepsilon}=\left(\left|\Phi^{n+1}\right|^{2}+\varepsilon\right)^{1 / 2}$ and to proceed as before. Following the same computation as that one of the Lemma 2 we have 


$$
g_{\varepsilon} \Delta g_{\varepsilon}=\left|\nabla\left(\Phi^{n+1}\right)\right|^{2}-\left.\left.\frac{1}{4}\left(\left|\Phi^{n+1}\right|^{2}+\varepsilon\right)^{-1}|\nabla| \Phi^{n+1}\right|^{2}\right|^{2}+\left\langle\Delta\left(\Phi^{n+1}\right), \Phi^{n+1}\right\rangle .
$$

On the other hand a similar result like that one of Lemma 1 is also true for $\Phi^{n+1}$, that is,

$$
\left.\left.|\nabla| \Phi^{n+1}\right|^{2}\right|^{2} \leq \frac{4 n}{(n+2)}\left|\Phi^{n+1}\right|^{2}\left|\nabla\left(\Phi^{n+1}\right)\right|^{2} .
$$

From (3.11) and (3.12) it follows that

$$
g_{\varepsilon} \Delta g_{\varepsilon} \geq \frac{2\left(\left|\Phi^{n+1}\right|^{2}+\varepsilon\right)^{-1}}{(n+2)}\left|\Phi^{n+1}\right|^{2}\left|\nabla\left(\Phi^{n+1}\right)\right|^{2}+\left\langle\Delta\left(\Phi^{n+1}\right), \Phi^{n+1}\right\rangle .
$$

Since $\operatorname{tr} H_{n+1}=\sum_{i} h_{i i}^{n+1}=n H$ and $H$ is constant we have $\sum_{i} \Delta h_{i i}^{n+1}=0$. This yields

$$
\sum_{i, j} h_{i j}^{n+1} \Delta h_{i j}^{n+1}=\sum_{i, j} \Phi_{i j}^{n+1} \Delta \Phi_{i j}^{n+1}+H \sum_{i} \Delta h_{i i}^{n+1}=\left\langle\Delta\left(\Phi^{n+1}\right), \Phi^{n+1}\right\rangle .
$$

We use also the following inequality obtained by Z. Hou $([\mathrm{H}]$, p. 39)

$$
\sum_{i, j} h_{i j}^{n+1} \Delta h_{i j}^{n+1} \geq n\left|\Phi^{n+1}\right|^{2}\left(1-\frac{|A|^{2}}{2 \sqrt{n-1}}\right) .
$$

From (3.13), (3.14) and (3.15) we have

$$
g_{\varepsilon} \Delta g_{\varepsilon} \geq\left|\Phi^{n+1}\right|^{2}\left(\frac{2\left(\left|\Phi^{n+1}\right|^{2}+\varepsilon\right)^{-1}}{(n+2)}\left|\nabla\left(\Phi^{n+1}\right)\right|^{2}+n-\frac{n|A|^{2}}{2 \sqrt{n-1}}\right) .
$$

Since $g_{\varepsilon} L_{3} g_{\varepsilon}=-g_{\varepsilon} \Delta g_{\varepsilon}-(n / 2 \sqrt{(n-1)})|A|^{2} g_{\varepsilon}^{2}$ we obtain

$$
\begin{aligned}
g_{\varepsilon} L_{3} g_{\varepsilon} \leq & -n\left|\Phi^{n+1}\right|^{2}+\frac{n|A|^{2}\left|\Phi^{n+1}\right|^{2}}{2 \sqrt{n-1}}-\frac{n|A|^{2}}{2 \sqrt{n-1}}\left(\left|\Phi^{n+1}\right|^{2}+\varepsilon\right) \\
& -\frac{2\left(\left|\Phi^{n+1}\right|^{2}+\varepsilon\right)^{-1}}{(n+2)}\left(\left|\Phi^{n+1}\right|^{2}\left|\nabla\left(\Phi^{n+1}\right)\right|^{2}\right)
\end{aligned}
$$

that is,

$$
g_{\varepsilon} L_{3} g_{\varepsilon} \leq-n\left|\Phi^{n+1}\right|^{2}-\frac{2\left(\left|\Phi^{n+1}\right|^{2}+\varepsilon\right)^{-1}}{(n+2)}\left(\left|\Phi^{n+1}\right|^{2}\left|\nabla\left(\Phi^{n+1}\right)\right|^{2}\right) .
$$

Since $M^{n}$ is not pseudo-umbilical $\lim _{\varepsilon \rightarrow 0} \int_{M} g_{\varepsilon}^{2} * 1=\int_{M}\left|\Phi^{n+1}\right|^{2}>0$. Therefore using again the characterization of $\mu_{1}$ given by Rayleigh quotient we obtain

$$
\mu_{1} \leq-\frac{2}{(n+2)} \frac{\int_{M}\left|\Phi^{n+1}\right|^{2}\left(n(n+2) / 2+\left(\left|\Phi^{n+1}\right|^{2}+\varepsilon\right)^{-1}\left|\nabla\left(\Phi^{n+1}\right)\right|^{2}\right) * 1}{\int_{M}\left(\left|\Phi^{n+1}\right|^{2}+\varepsilon\right) * 1} .
$$

Making $\varepsilon \rightarrow 0$ in the last inequality we have 


$$
\mu_{1} \leq-n-\frac{2}{(n+2)} \frac{\int_{M}\left|\nabla\left(\Phi^{n+1}\right)\right|^{2} * 1}{\int_{M}\left|\Phi^{n+1}\right|^{2} * 1},
$$

which concludes the proof of the proposition.

We consider now the case when $M^{n}$ is pseudo-umbilical and has codimension $p \geq 2$. Introducing the Schrödinger operator $L_{4}=-\Delta-(3 / 2)|\Phi|^{2}$ we derive the following proposition.

Proposition 3. Let $M^{n}$ be a closed submanifold immersed in $S^{n+p}$ such that $M^{n}$ is pseudo-umbilical with parallel mean curvature vector $h$. If $M^{n}$ is not totally umbilic, $p \geq 2$ and $\mu_{1}$ is the first eigenvalue of $L_{4}$, then

$$
\mu_{1} \leq-n\left(1+H^{2}\right)-\frac{2}{(n+2)} \frac{\int_{M}|\nabla \Phi|^{2} * 1}{\int_{M}|\Phi|^{2} * 1} .
$$

Proof. Taking into account that $M^{n}$ is pseudo-umbilical we may use the following inequality due to Hou $([\mathrm{H}]$, p. 42$)$

$$
\langle\Delta \Phi, \Phi\rangle \geq|\Phi|^{2}\left(n\left(1+H^{2}\right)-\frac{3}{2}|\Phi|^{2}\right) .
$$

Therefore considering again $f_{\varepsilon}=\left(|\Phi|^{2}+\varepsilon\right)^{1 / 2}$ the Lemma 2 yields

$$
f_{\varepsilon} \Delta f_{\varepsilon} \geq|\Phi|^{2}\left(\frac{2\left(|\Phi|^{2}+\varepsilon\right)^{-1}|\nabla \Phi|^{2}}{(n+2)}+n\left(1+H^{2}\right)-\frac{3}{2}|\Phi|^{2}\right) .
$$

Since $f_{\varepsilon} L_{4} f_{\varepsilon}=-f_{\varepsilon} \Delta f_{\varepsilon}-(3 / 2)|\Phi|^{2} f_{\varepsilon}^{2}$ we get

$$
f_{\varepsilon} L_{4} f_{\varepsilon} \leq-n\left(1+H^{2}\right)|\Phi|^{2}-\frac{2\left(|\Phi|^{2}+\varepsilon\right)^{-1}}{(n+2)}|\Phi|^{2}|\nabla \Phi|^{2} .
$$

On the other hand since $M^{n}$ is not totally umbilic we have

$$
\lim _{\varepsilon \rightarrow 0} \int_{M} f_{\varepsilon}^{2} * 1=\int_{M}|\Phi|^{2} * 1>0 .
$$

Hence we may use $f_{\varepsilon}$ as a test function to estimate $\mu_{1}$. Taking into account that $\mu_{1} \leq \int_{M} f_{\varepsilon} L_{4} f_{\varepsilon} * 1 / \int_{M} f_{\varepsilon}^{2} * 1$ and $H$ is constant we derive from (3.17) that

$$
\mu_{1} \leq-n\left(1+H^{2}\right)-\frac{2}{(n+2)} \frac{\int_{M}|\nabla \Phi|^{2} * 1}{\int_{M}|\Phi|^{2} * 1},
$$

which completes the proof of the Proposition 3.

We point out now that to derive the Theorem 2 it is enough to apply the Proposition 1 with the result obtained independently by W. Santos and $\mathrm{H}$. Xu. In fact, from that proposition we get $\mu_{1}=0$ if, and only if, $M^{n}$ is totally umbilic, 
otherwise $\mu_{1} \leq-n\left(1+H^{2}\right)$. Suppose now that $|\Phi|^{2}=\rho \neq 0$. Then $L_{2}=-\Delta-$ $n\left(1+H^{2}\right)$ and $\mu_{1}=-n\left(1+H^{2}\right)$. On the other hand, it follows again from Proposition 1 that $|\nabla \Phi|=0$ provided $\mu_{1}=-n\left(1+H^{2}\right)$. Therefore $\Phi$ and $\left|\Phi_{h}\right|$ are constants. Hence we conclude that

$$
\mu_{1}=-\left(B_{p, h}\right)^{-1}|\Phi|^{2}-\frac{n(n-2)}{\sqrt{n(n-1)}}\left|\Phi_{h}\right| .
$$

From where we have $|\Phi|^{2}=\rho$. In order to complete the rest of the proof of the theorm we may apply a theorem due to Santos ([S], p. 405) or Xu ([X], p. 494) presented in the introduction that describes all submanifolds $M^{n}$ immersed in the Euclidean sphere $S^{n+p}$ with parallel mean curvature vector $h$ and $|\Phi|^{2}=\rho$. More precisely, they have proved:

If $\rho=0$ then $M^{n}$ is a sphere, otherwise $M^{n}$ is either one of the Clifford tori or one of the Veronese surfaces in $S^{n+p}$.

\section{Applications}

In this section we will present two applications of our main theorem. We point out now that $|\Phi|^{2}=\rho$ has two main consequences: either $L_{2}=-\Delta$, or $L_{2}=-\Delta-n\left(1+H^{2}\right)$. In fact, the former case comes from $\rho=0$, whereas the last one comes from $\rho \neq 0$. Hence, we may derive from the theorem due to Santos $([\mathrm{S}])$ or $\mathrm{Xu}([\mathrm{X}])$ and the Theorem 2 the following theorem:

THEOREM 3. Let $M^{n}$ be a closed submanifold of $S^{n+p}, p \geq 2$, with non null parallel mean curvature vector $h$ and let $L_{3}=-\Delta-\left(n|A|^{2} / 2 \sqrt{n-1}\right)$ be the operator with first eigenvalue $\mu_{1}$. If $n \geq 3$ and $M^{n}$ is not pseudo-umbilical then $\mu_{1} \leq-n$. Moreover, $\mu_{1}=-n$ if, and only if, $M^{n}$ is the Clifford torus $S^{1}(r) \times$ $S^{n-1}(s) \hookrightarrow S^{n+1} \hookrightarrow S^{n+p}$, with $s^{2}=\sqrt{n-1}(1+\sqrt{n-1})^{-1}$ and $r^{2}=(1+\sqrt{n-1})^{-1}$. If $n=2$ then $M^{2}$ is a totally umbilical sphere $S^{2}\left(1 /\left(1+H^{2}\right)\right)$.

Proof. By using Proposition 2 we infer that if $M^{n}$ is not pseudoumbilical then $\mu_{1} \leq-n$. We note that $|A|^{2}=2 \sqrt{n-1}$ implies $L_{3}=-\Delta-n$. From where we conclude $\mu_{1}=-n$. Conversely, if $\mu_{1}=-n$, Proposition 2 shows that $\left|\nabla\left(\Phi^{n+1}\right)\right|=0$. Hence $\left|\Phi^{n+1}\right|^{2}$ and $\left|A^{n+1}\right|^{2}=\left|\Phi^{n+1}\right|^{2}+n H^{2}$ are constants. Using (3.15) and the assumption $\left|A^{n+1}\right|^{2}$ is constant we derive

$$
|A|^{2} \geq 2 \sqrt{n-1} \text {. }
$$

In fact, since $(1 / 2) \Delta\left|A^{n+1}\right|^{2}=\sum_{i, j, k}\left(h_{i j k}^{n+1}\right)^{2}+\sum_{i, j} h_{i j}^{n+1} \Delta h_{i j}^{n+1}$ we have

$$
0=\sum_{i, j, k}\left(h_{i j k}^{n+1}\right)^{2}+\sum_{i, j} h_{i j}^{n+1} \Delta h_{i j}^{n+1} \geq n\left|\Phi^{n+1}\right|^{2}\left(1-\frac{|A|^{2}}{2 \sqrt{n-1}}\right),
$$

which gives the desired inequality. 
On the other hand if $\Gamma=\left\{f \in C^{\infty}(M): f \neq 0\right\}$ then Rayleigh quotient yields $\mu_{1}=\inf _{f \in \Gamma}\left(\int_{M} f L_{3} f * 1 / \int_{M} f^{2} * 1\right)$. Since $|A|^{2} \geq 2 \sqrt{n-1}$ and $\mu_{1}=-n$ we conclude that $|A|^{2}=2 \sqrt{n-1}$. Thus, if $n \geq 3$ then $M^{n}$ is isometric to a Clifford torus according to a result due to Hou ([H], p. 40).

For $n=2$ the same result yields that $M^{2}$ is a totally umbilical sphere in $S^{2+p}$. This completes the proof of the theorem.

Finally we treat the case when $M^{n}$ is pseudo-umbilical with parallel mean curvature vector. More precisely we have the following theorem.

THEOREM 4. Let $M^{n}$ be a closed submanifold immersed in $S^{n+p}$ with parallel mean curvature vector $h$ and $p \geq 2$. Suppose in addition that $M^{n}$ is also pseudo-umbilical and let $\mu_{1}$ be the first eigenvalue of $L_{4}=-\Delta-(3 / 2)|\Phi|^{2}$. If $M^{n}$ is totally umbilical, then $\mu_{1}=0$. Otherwise $\mu_{1} \leq-n\left(1+H^{2}\right)$. Furthermore, if $\mu_{1}=-n\left(1+H^{2}\right)$ we have: a) Either $M^{n}$ is the Clifford torus

$$
S^{k}(r) \times S^{n-k}(s) \hookrightarrow S^{n+1}\left(\frac{1}{\sqrt{1+H^{2}}}\right) \hookrightarrow S^{n+2} \hookrightarrow S^{n+p}
$$

b) Or else, $M^{n}$ is the Veronese surface $M^{2} \hookrightarrow S^{4}\left(1 / \sqrt{1+H^{2}}\right) \hookrightarrow S^{5} \hookrightarrow S^{n+p}$.

Proof. From Proposition 3 we get $\mu_{1}=0$ if, and only if, $M^{n}$ is totally umbilic, otherwise $\mu_{1} \leq-n\left(1+H^{2}\right)$. Now suppose $|\Phi|^{2}=(2 / 3) n\left(1+H^{2}\right)$, then the operator $L_{3}$ becomes $L_{3}=-\Delta-n\left(1+H^{2}\right)$ while $\mu_{1}=-n\left(1+H^{2}\right)$. Conversely, if $\mu_{1}=-n\left(1+H^{2}\right)$ it follows from Proposition 3 that $|\nabla \Phi|^{2}=0$. Hence $\Phi$ is constant, and so, in view of (3.16) we obtain $|\Phi|^{2}=(2 / 3) n\left(1+H^{2}\right)$. Now the conclusion of the theorem is a consequence of the Proposition 2 of $\mathrm{Z}$. Hou ([H], p. 42).

Acknowledgement. The authors would like to thank the referee for many valuable suggestions.

\section{REFERENCES}

[AC] Alencar, H. and do Carmo, M., Hypersurfaces with constant mean curvature in spheres, Proc. AMS, 120 (1994), 1223-1228.

[CdCK $]$ Chern, S. S., do Carmo, M. and Kobayshi, S., Minimal submanifolds of a sphere with second fundamental form of constant length, Functional Analysis and Related Fields, Edited by F. Browder, Springer, (1970), 393-409.

$[\mathrm{H}]$ Hou, Z., A pinching problem on submanifolds with parallel mean curvature vector field in a sphere, Kodai Math. Jour., 21 (1998), 35-45.

[L] Lawson, H. B., Local rigidity for minimal hypersurfaces, Ann. of Math., 89 (1969), 187197.

[S] Santos, W., Submanifolds with parallel mean curvature vector in spheres, Tôhoku Math. Jour., 46 (1994), 403-415.

[Si] Simons, J., Minimal varieties in Riemannian manifolds, Ann. of Math., 88 (1968), 62-105. 
[W] Wu, C., New characterizations of the Clifford tori and the Veronese surface, Arch. Math., 61 (1993), 277-284.

[X] Xu, H., A rigidity theorem for submanifolds with parallel mean curvature in a sphere, Arch. Math., 61 (1993), 498-496.

Departamento de Matemática

UFC-FoRTALEZA-CE-BR-60455-760

e-mail: abbarros@mat.ufc.br

http://www.mat.ufc.br/posgrad.html/

Departamento de Matemática

UFC-FoRTALEZA-CE-BR-60455-760

e-mail: aldir@mat.ufc.br

http://www.mat.ufc.br/posgrad.html/

Departamento de Matemática

E EsTATÍSTICA-UNIRIO-Rio

DE JANEIRO-RJ-BR-22290-240

e-mail: amancio@impa.br 